COLO-HEP-357, OU-HET 209

hep-th/9504033

March 1995

\title{
Thermodynamics of Quantum Fields in Black Hole Backgrounds
}

\author{
S. P. de Alwis' \\ Department of Physics, Box 390, University of Colorado, Boulder, CO 80309 \\ N. Ohtaf \\ Department of Physics, Osaka University, Toyonaka, Osaka 560, Japan
}

\begin{abstract}
We discuss the relation between the micro-canonical and the canonical ensemble for black holes, and highlight some problems associated with extreme black holes already at the classical level. Then we discuss the contribution of quantum fields and demonstrate that the partition functions for scalar and Dirac (Majorana) fields in static space-time backgrounds, can be expressed as functional integrals in the corresponding optical space, and point out that the difference between this and the functional integrals in the original metric is a Liouville-type action. The optical method gives both the correction to the black hole entropy and the bulk contribution to the entropy due to the radiation, while (if the Liouville term is ignored) the conical singularity method just gives the divergent contribution to the black hole entropy. A simple derivation of a general formula for the free energy in the high-temperature approximation is given and applied to various cases. We conclude with a discussion of the second law.
\end{abstract}

\footnotetext{
${ }^{1}$ e-mail: dealwis@gopika.colorado.edu

${ }^{2}$ e-mail: ohta@phys.wani.osaka-u.ac.jp
} 


\section{Introduction}

There has been renewed interest in the entropy of the black holes and its connection with the puzzle of information loss. If a black hole is formed from a pure quantum state and decays by emitting Hawking radiation in a thermal state in accordance with Hawking's "semi-classical" argument [1], it appears that a pure state evolves into a mixed state in contradiction to one of the fundamental laws of quantum mechanics, and quantum mechanical information is lost. This is quantified by the entanglement entropy of the final (mixed) state. There is also the related (though not identical) question of the validity of the second law of thermodynamics in semi-classical black hole physics.

The two issues mentioned above are actually related to two different concepts of entropy. What is relevant for the second question is the thermodynamic or coarse grained entropy, and applies even for classical black holes. This is associated with a description of the system that only specifies its macroscopic characteristics. It is this entropy that is expected to satisfy the second law of thermodynamics and is given according to Boltzmann by the logarithm of the available phase space. This concept can be extended to quantum mechanics where the phase space volume (or rather the number of phase space cells) would have to be replaced by the dimension of the Hilbert space available to the system (the two being the same in the semi-classical limit). However it is difficult to understand how the Bekenstein-Hawking entropy of a black hole [2, 3] which is proportional to the area of the global horizon $\left(S_{B H}=\frac{A}{4 G_{N} \hbar}\right)$ could be interpreted as the Boltzmann entropy since the latter is expected to be an extensive quantity.2]

On the other hand, in quantum mechanics there is another concept of entropy which is applicable when a system is described in terms of a set of (commuting) dynamical variables which are not complete. Typically this arises when the object under study is part of a larger system (which may be in a pure state) and therefore has to be described

\footnotetext{
${ }^{1}$ As usual the ultraviolet catastrophe has to be regulated by introducing $\hbar$; i.e. the Boltzmann entropy of a system for which the available phase space volume is $\Gamma$ has the value $S=\ln \frac{\Gamma}{h^{N}}$, N being half the dimension of the phase space.

${ }^{2}$ See ref. [4], however.
} 
by a density matrix $\rho$ obtained by tracing over all the variables of the rest of the large system. The microscopic entropy is then defined to be $S=-\operatorname{tr} \rho \ln \rho$. This entropy arises because of the correlations between the states of the object and the states of the environment. In quantum field theory this entropy may be associated with the fields inside a region of space which are correlated with the fields outside. The system is then the field configuration in the region in question and the term geometric entropy has been used for its microscopic entropy. It has been argued [5, [6] that (at least in flat space) the geometric entropy is proportional to the area. This is plausible because of the result that the entropy in the interior of the region is exactly equal to the entropy of the outside so that each must be dependent on the common boundary [6]. Clearly if these arguments can be extended to black hole space-time, then one could have a statistical mechanics basis for the Bekenstein-Hawking entropy at least in the case where the Einstein term is induced by quantum fluctuations like in string theory. Some steps in this direction have recently appeared in the literature [7, 8]. In particular, a functional integral expression for the geometric entropy of Rindler space has been given in [8]. On the other hand, Ref. [7]

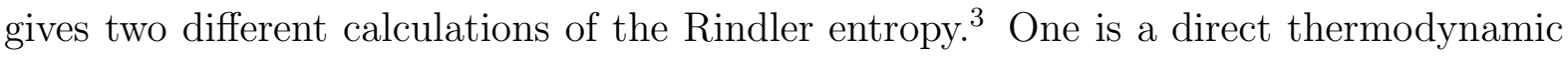
(Hamiltonian) calculation of the thermal entropy of a gas of bosons in Rindler space. The other is a calculation of the path integral representation of the thermal partition function as in [8]. The calculations of the entropy in both these cases agree. However the free energy has different values. The path integral expression is of course the same in both [7] and [8] and both give a vanishing free energy at the Rindler temperature. On the other hand, the direct thermodynamic calculation gives a non-zero value. One of the purposes of this work is to investigate this difference. It will be found that it arises from the different measures that one has to use for the thermal partition function and the geometric one.

The relevance of these works to the black hole is that the Rindler space is the limit of infinite black hole mass in a Schwarzschild space-time. However it is clearly desirable to

\footnotetext{
${ }^{3}$ In all of these calculations the entropy per unit area on the Rindler horizon is a constant in agreement with the expectations for geometric entropy.
} 
study the finite mass case. In particular one would like to distinguish between the two types of entropy. We expect that the geometric entropy is proportional to the area of the horizon and there should be no bulk term. On the other hand, the thermal entropy should presumably have a bulk term corresponding to the gas of particles (quantum fields) outside the black hole as well as an area term, i.e. a correction to the black hole entropy. Now if one considers a series of quasi-static frames corresponding to black holes of decreasing mass, one should find that the microscopic entropy decreases to zero. This entropy is divergent because of correlations between fields which are arbitrarily close to the horizon on either side of it [9]. Thus this procedure requires one to impose a cut off at the horizon that is kept fixed in every frame. Alternatively one has to consider a theory with a natural short distance cutoff (e.g. string theory) as advocated by Susskind and Uglum [7]. If the area law is a measure of the information hidden behind the horizon, clearly it must come out in the Hawking radiation as the black hole decays. Thus it seems very important to establish the area law for the geometric entropy in the case of a finite mass black hole. Unfortunately there is no straightforward generalization of the Rindler space arguments of [8].

We consider next the canonical ensemble for quantum fields in a given static background. It is shown that what one obtains for the partition function is a Euclidean path integral but the relevant metric is not the original Rindler or black hole one but is the so-called optical metric introduced by Gibbons and Perry [10]. The optical metric is conformally related to the original (Rindler or black hole) metric and our expression differs from those in the geometric formulation by an action analogous to the Liouville action. For instance in two-dimensional Rindler space, we show that this partition function is the same as that considered by Callan and Wilczek [8 except for the well-known Liou-

\footnotetext{
${ }^{4}$ This metric was used in connection with this problem many years ago by Dowker and Kennedy [11] who also seem to have been the first to derive the high-temperature asymptotic expansion given below (3.26). However these authors did not discuss the divergences in the free energy and entropy arising from the divergence of the optical volume. The metric has also been used recently by [12, 13. However there was no discussion of the relation of the optical method to the conical singularity one in the original metric, in any of these papers.
} 
ville action. It is this Liouville term which gives a non-zero free energy to Rindler space (at the Rindler temperature $T=1 / 2 \pi$ ). In the four-dimensional case (even for Rindler space) the partition function cannot be calculated exactly. However in any dimension and for any static backgrounds, we can obtain a formula for the free energy (and hence the entropy) in the high-temperature approximation.

Unlike what one expects for the microscopic entropy, for our thermodynamic entropy one obtains also a bulk contribution representing the free energy of a gas of particles as well as terms which depend on the mass of the black hole and which would go to zero as the black hole decays. These terms enable us to discuss the operation of the second law. It should be stressed again that this applies to the thermodynamic entropy and not to the entanglement (geometric) entropy.

In four-dimensional black hole backgrounds, we find that in addition to the linear divergence in the entropy (and free energy), there is also a logarithmic divergence. Thus renormalization of $G_{N}$ alone is not sufficient to make the entropy finite. One also needs to introduce a bare $R^{2}$ term to the original action to deal with this.? We also investigate the entropy of quantum fields around Reissner-Nordström and dilaton black holes. In the former case, we find that there is a cubic order divergence as the extreme limit is approached. This is present in addition to the linear (quadratic in terms of the proper cutoff) and logarithmic divergences of the Schwarzschild case which can be incorporated into the renormalization of Newton's constant [7] and coefficients of higher derivative terms in the effective action. We believe that this signifies a break down of the thermal ensemble for these extreme holes. For the dilaton black hole, on the other hand, we find that the free energy has a linear as well as a logarithmic divergence. The former is zero (as is the "classical" entropy), but the latter is non-zero in the extreme limit. A short account of these calculations was presented by us in [15]. The calculations for the dilaton and Reissner-Nordström black holes have also been done by a different method in [16], with results in agreement with ours.

\footnotetext{
${ }^{5}$ This has already been pointed out in 14 .
} 


\section{The "Classical" Entropy of Black Holes}

We begin with the micro-canonical formulation of (quantum) statistical mechanics. Introducing the Hamiltonian operator $H$ of the system and its eigenstates $|E, r\rangle$ $(H|E, r\rangle=E|E, r\rangle)$ where $r$ labels the eigenvalues of all other commuting observables which serve to characterize the quantum state. The degeneracy of the energy eigenstates then gives us a definition of the entropy. Writing $N(E)=\operatorname{tr} \delta(E-H)$, we define the entropy as the logarithm of this degeneracy,

$$
S=\ln N(E)
$$

The (inverse) temperature is then defined by

$$
T^{-1}=\frac{\partial S}{\partial E}
$$

In taking this formalism over to discuss the thermodynamics of space-time itself, the first problem one faces is the definition of energy. As is well known, one may define an energy when the space-time is asymptotically flat. Thus we define the quantum mechanics of metrics and other fields by (say) a functional integral formulation in which the fields die away asymptotically (in the space variables).

In order to calculate this entropy in quantum field theory, one needs to introduce the Laplace transform of the degeneracy $N(E)$, i.e. the so-called partition function:

$$
Z(\beta)=\int d E e^{-\beta E} N(E)=\int d E e^{-\beta E} \operatorname{tr} \delta(E-H)=\operatorname{tr} e^{-\beta H} .
$$

It should be stressed that here $\beta$ is just the Laplace transform variable and we do not necessarily have to give it a physical interpretation as an inverse temperature. The latter will be the case in a rather different context where we have the canonical ensemble with the system being in equilibrium with a heat bath.

By standard arguments, we obtain

$$
Z(\beta)=\int_{\beta}[d g][d \phi] e^{-I_{g}^{\beta}-I_{m}^{\beta}}
$$

\footnotetext{
${ }^{6}$ The micro-canonical ensemble for black holes is discussed in some detail in [17].
} 
where

$$
I_{g}^{\beta}=-\frac{1}{16 \pi} \int_{\mathcal{M}_{\beta}} R-\frac{1}{8 \pi} \int_{\partial \mathcal{M}} K,
$$

and $I_{m}^{\beta}$ are the Euclidean gravitational and matter actions over a space-time manifold $\mathcal{M}_{\beta}$ which has toroidal topology in the (Euclidean) time direction with period $\beta$. The subscript on the functional integral is an instruction to impose these boundary conditions on the fields. In the next section, it will be shown that the measure in the functional integration is not the naive measure but this fact is irrelevant for our "classical" considerations in this section.

In order to evaluate this in the saddle point approximation, one needs the classical action. This has the value [3, 18 (assuming that the dominant contribution to the functional integral coming from the matter action is the zero field one)

$$
I=\beta M-\frac{A}{4}=\beta M-4 \pi M^{2} .
$$

The first term is simply the value of the classical Hamiltonian multiplied by the Euclidean time interval $\beta$, and the second term is quarter the area of the horizon for black hole solutions, which we have set equal to its value for the Schwarzschild solution in the second equality. $M$ of course is an integration constant that is equal to the value of the boundary Hamiltonian i.e. the mass of the space-time. Now usually in the literature this value is substituted into the functional integral, thus approximating it by its classical value of the integrand at some fixed $M$. However it is more appropriate to integrate over $M$ since one has an integral over all metrics. Indeed that is precisely what one should expect from a partition function i.e. a function of a variable $\beta$ that is conjugate to the energy! Thus our approximate evaluation of the partition function gives, (restricting ourselves to Schwarzschild spaces)

$$
Z \simeq \int d M e^{-\beta M+4 \pi M^{2}}
$$

This integral is of course divergent but the important point is that this divergence has a physical interpretation. Comparing with (2.3), we see that $N(E)=e^{\frac{A}{4}}=e^{4 \pi E^{2}}$ so that from (2.1) and (2.2) we get the Bekenstein-Hawking entropy and inverse Hawking 
temperature

$$
S=\frac{A}{4}=4 \pi E^{2}, \quad T_{H}^{-1} \equiv \beta_{H}=8 \pi E .
$$

The free energy of the space may then be defined in the usual manner and we get $F=$ $E-T_{H} S=\frac{E}{2}$.

Similarly for Reissner-Nordström black holes, $S=\frac{A}{4}=\pi r_{+}^{2}$ where $r_{+}$is the radius of the outer horizon $\left(r_{ \pm}=E \pm \sqrt{E^{2}-Q^{2}}\right)$ and $Q$ is the charge that is kept fixed as an external macroscopic parameter, like the energy $E$ of the black hole. From (2.2), the Hawking temperature is then $T=\frac{\left(r_{+}-r_{-}\right)}{4 \pi r_{+}^{2}}$. In the extreme case $E \rightarrow Q$ this tends to zero and the entropy $\rightarrow \pi E^{2}$. On the other hand, one may discuss the thermodynamics of the extreme holes without reference to the non-extreme case. In this case we have for the action $I=\beta M$ (see third paper of [3] and [18]) and the partition function is

$$
Z=\int e^{-\beta M} d M=\beta^{-1}
$$

Comparing this with (2.3), we find $N(E)=1$ i.e. $S=0$. Hence $T^{-1}=\frac{\partial S}{\partial E}=0$. In other words, the Hawking temperature is infinite! On the other hand, purely geometric arguments seem to indicate that the Hawking temperature is arbitrary (see third paper of [3] and [18]). Basically the reason is that the topology for the extreme hole is completely different from that for the non-extreme case and it has no conical singularity at any temperature. These ambiguities suggest that the statistical mechanics of these objects is not well-defined. Further evidence of this will be given in sect. 4 when we compute the quantum corrections to these classical values.

To summarize then, in this micro-canonical calculation the partition function need not be given physical significance. It is merely a calculational device enabling us to use the path integral formulation of quantum field theory. The corresponding $\beta$ is just the Laplace transform variable and need not be interpreted as the inverse temperature of the system. The entropy and physical inverse temperature are calculated from (2.1, 2.2), and yield the well-known Bekenstein-Hawking and Hawking results, respectively.

The canonical emsemble, on the other hand, is not well-defined for black holes since the partition function is divergent. Note that unlike the partition function for strings 
which diverges only above the Hagedorn temperature, in our case it is divergent at all "temperatures" since the degeneracy grows as $e^{E^{2}}$ rather than $e^{E}$. Nevertheless one may make some formal arguments. If one makes the saddle point approximation in (2.7), we

get $Z=e^{-\frac{\beta^{2}}{16 \pi}}$. This gives an average value for the black hole mass $\langle M\rangle=\frac{\beta}{8 \pi}$. This is the same relation as in the micro-canonical ensemble but now with the average mass of the thermal ensemble replacing the actual mass. Thus we have agreement between the micro-canonical and the canonical ensembles for the non-extreme Reissner-Nordström black hole, but for the extreme case (where $Z$ is actually well-defined) we have from (2.9), $\langle M\rangle=-\frac{\partial \ln Z}{\partial \beta}=\beta^{-1}$. Similarly the entropy evaluated using the canonical ensemble formula gives $S=-\left(\beta \frac{\partial}{\partial \beta}-1\right) \ln \beta=-1+\ln \beta$. These relations are not in agreement either with the results of the micro-canonical calculation which gave an infinite temperature and zero entropy nor with the geometric calculation which gave an arbitrary temperature and zero entropy. There seems to be further evidence that the thermodynamics of these extreme objects (if it is at all meaningful) is somewhat strange. Later we will suggest a reason for this peculiar thermodynamics (see discussion after equation (6.7)).

\section{Free Energy of Scalar Fields}

We wish to derive a functional integral expression for the thermal ensemble for a free scalar field in a static background with metric.

$$
d s^{2}=g_{00} d t^{2}+h_{i j} d x^{i} d x^{j}
$$

We write $g=\operatorname{det} g_{\mu \nu}=g_{00} h, h=\operatorname{det} h_{i j}$ where $\mu, \nu=0, \ldots, D-1 ; i, j=1, \ldots D-1$. The action is

$$
\begin{aligned}
S & =-\frac{1}{2} \int d^{D} x \sqrt{-g} g^{\mu \nu} \partial_{\mu} \phi \partial_{\nu} \phi \\
& =\int d t \int d^{D-1} x \sqrt{h}\left[\frac{1}{2 \sqrt{-g_{00}}} \dot{\phi}^{2}-\frac{\sqrt{-g_{00}}}{2} h^{i j} \partial_{i} \phi \partial_{j} \phi\right] .
\end{aligned}
$$

\footnotetext{
${ }^{7}$ We are ignoring graviton fluctuations. These are technically more complicated but we do not expect them to change the qualitative physics.
} 
The canonical momentum is $\pi=\frac{\dot{\phi}}{\sqrt{-g_{00}}}$ and the Hamiltonian is

$$
H=\int d^{D-1} x \mathcal{H}=\int d^{D-1} x \sqrt{h} \sqrt{-g_{00}}\left[\frac{1}{2} \pi^{2}+\frac{1}{2} h^{i j} \partial_{i} \phi \partial_{j} \phi\right] .
$$

The equal-time canonical commutation relations are

$$
[\hat{\phi}(\vec{x}), \hat{\pi}(\vec{y})]=\frac{i}{\sqrt{h}} \delta(\vec{x}-\vec{y}) .
$$

We introduce a basis of eigenstates of the field operator $\hat{\phi}$ which are (delta function) orthonormal and complete on the space of fields with metric $\|\delta \phi\|^{2}=\int d^{D-1} x \sqrt{h}(\delta \phi)^{2}$. Similarly we may introduce a basis of eigenstates of the canonical momentum operator $\pi$. Using the transformation matrix $\langle\phi \mid \pi\rangle=\frac{1}{\sqrt{2 \pi}} e^{i \int d^{D-1} \sqrt{h} \phi \pi}$ we may then obtain from the usual time-slicing procedure the following expression for the partition function:

$$
\operatorname{Tr}\left[e^{-\beta H}\right]=\int[d \pi] \int_{\phi(0, \vec{x})=\phi(\beta, \vec{x})}[d \phi] e^{-\int_{0}^{\beta} d t \int d^{D-1} x \sqrt{h}[-i \pi \dot{\phi}+\mathcal{H}]},
$$

where $\mathcal{H}=\sqrt{-g_{00}}\left[\frac{\pi^{2}}{2}+\frac{1}{2} h^{i j} \partial_{i} \phi \partial_{j} \phi\right]$.

The Gaussian integral over $\pi$ gives the factor $\Pi_{t, \vec{x}} \frac{1}{\left(-g_{00}\right)^{\frac{1}{4}}} e^{-\int_{0}^{\beta} d t \int d^{D-1} \sqrt{h} \frac{\dot{\phi}^{2}}{2 \sqrt{-g_{00}}}}$ and we finally have the expression

$$
\int_{\phi(0, \vec{x})=\phi(\beta, \vec{x})} \prod_{t, \vec{x}} d \phi\left(\frac{h}{g_{00}^{E}}(t, \vec{x})\right)^{\frac{1}{4}} e^{-\int_{0}^{\beta} d t \int d^{D-1} x \sqrt{g^{E}} \frac{1}{2} g^{E, \mu \nu} \partial_{\mu} \phi \partial_{\nu} \phi},
$$

where $g_{\mu \nu}^{E}=\left(-g_{00}, h_{i j}\right)$ is the Euclideanized metric. Note in the above that although the action in the exponent of the functional integral is covariant, the measure is not. This is a reflection of the fact that a particular $3+1$ split is used in defining the partition function. Henceforth we will work with the Euclidean metric and the superscript $E$ will be omitted.

It is convenient to discuss conformally coupled scalars and to introduce a mass term, so we will change the matter action (after partial integration) to

$$
S_{\phi}=\int_{0}^{\beta} d t d^{D-1} x \sqrt{g} \phi\left(K+m^{2}\right) \phi
$$

where

$$
K \equiv-\square+\frac{1}{4} \frac{D-2}{D-1} R, \square \equiv \frac{1}{\sqrt{g}} \partial_{\mu}\left(\sqrt{g} g^{\mu \nu} \partial_{\nu}\right)
$$


Thus we may write

$$
\operatorname{Tr}\left[e^{-\beta H}\right]=\int_{\phi(0, \vec{x})=\phi(\beta, \vec{x})} \prod_{t, \vec{x}} d \phi \Omega g^{\frac{1}{4}}(t, \vec{x}) e^{-\int_{0}^{\beta} d t d^{D-1} x \sqrt{g} \phi\left(K+m^{2}\right) \phi} .
$$

In the above, $\Omega=\frac{1}{\sqrt{g_{00}}}$ is a conformal factor which causes a mismatch between the metric background of the action and that defining the functional integral. In order to take into account this mismatch, we use the optical metric (introduced in [10] for somewhat different reasons) and perform a change of field variable. Thus writing

$$
\bar{g}_{\mu \nu}=\Omega^{2} g_{\mu \nu}, \quad \bar{\phi}=\Omega^{\frac{2-D}{2}} \phi
$$

we have for the measure

$$
\prod_{t, \vec{x}} d \phi \Omega g^{\frac{1}{4}}(t, \vec{x})=\prod_{t, \vec{x}} d \bar{\phi} \bar{g}^{\frac{1}{4}}(t, \vec{x})
$$

Using also the properties of the Laplacian and the curvature under a conformal transformation (see for example [19]), we finally obtain

$$
\operatorname{Tr}\left[e^{-\beta H}\right]=\int_{\bar{\phi}(0, \vec{x})=\bar{\phi}(\beta, \vec{x})} \prod_{t, \vec{x}} d \bar{\phi} \bar{g}^{\frac{1}{4}}(t, \vec{x}) e^{-\int_{0}^{\beta} d t d^{D-1} x \sqrt{\bar{g}} \bar{\phi}\left(\bar{K}+m^{2} \Omega^{-2}\right) \bar{\phi}} .
$$

Note that the optical metric is of the form

$$
\bar{d} s^{2}=d t^{2}+\frac{h_{i j}}{g_{00}} d x^{i} d x^{j}
$$

and (since the original metric is static) the topology of optical space is $S^{1} \times \mathcal{M}^{D-1}$.

Now much of the recent discussion of black hole entropy has been carried out using the path integral in the original metric. So it is important to understand the relation of that to (3.12). Let us take the massless case. Then we have the well-known result,

$$
\int \prod_{t, \vec{x}} d \bar{\phi} \bar{g}^{\frac{1}{4}}(t, \vec{x}) e^{-\int_{0}^{\beta} d t d^{D-1} x \sqrt{g} \bar{\phi} \bar{K} \bar{\phi}}=\int \prod_{t, \vec{x}} d \phi g^{\frac{1}{4}}(t, \vec{x}) e^{-\int d^{D} x \sqrt{g} \phi K \phi+\Gamma[\Omega, g]},
$$

where $\Gamma$ is the Liouville-type action. In two dimensions this has the form [20]

$$
\Gamma[\Omega, g]=\frac{1}{24 \pi} \int \sqrt{g} g^{\mu \nu} \partial_{\mu} \sigma \partial_{\nu} \sigma
$$


while in four dimensions it is 21]

$$
\Gamma=\int d^{4} x \sqrt{g}\left[2 b^{\prime} \sigma \Delta_{4} \sigma+\left\{b F+b^{\prime}\left(G-\frac{2}{3} \square R\right)\right\} \sigma\right],
$$

where $\sigma=\ln \Omega, F$ is the square of the Weyl tensor, $G$ is the Euler density, $\Delta_{4}$ is a fourth order differential operator which also involves the Ricci tensor and scalar curvature of $g$. In eq. (3.16), $b, b^{\prime}$ are numerical coefficients whose values we do not need for the moment. Thus we have two different expressions for the free energy at an inverse temperature $\beta$ :

$$
\begin{aligned}
-\beta F & =-\frac{1}{2} \ln \operatorname{det}\left[K_{\beta}+m^{2}\right]+\Gamma[\Omega, g] \\
& =-\frac{1}{2} \ln \operatorname{det}\left[\bar{K}_{\beta}+m^{2} \Omega^{-2}\right] .
\end{aligned}
$$

The first line is the calculation in the original metric while the second line gives the optical metric version. The difference between the two determinants is the Liouville-type action (upto terms which vanish as $m \rightarrow 0$ ). The determinant on the first line has the heat kernel representation

$$
\ln \operatorname{det}\left[K_{\beta}+m^{2}\right]=-\int_{\epsilon}^{\infty} \frac{d s}{s} \int \sqrt{g} d^{D} x H(s \mid x, x),
$$

where the heat kernel is defined by $H\left(s \mid x, x^{\prime}\right)=e^{-s\left(K+m^{2}\right)} \frac{1}{\sqrt{g}} \delta^{D}\left(x-x^{\prime}\right)$ and $\epsilon$ is an ultraviolet cutoff. The trace of the heat kernel has the asymptotic expansion (see for example [19]) 8

$$
\int \sqrt{g} d^{D} x H(s \mid x, x)=(4 \pi s)^{-\frac{D}{2}} e^{-s m^{2}} \sum_{k=1}^{\infty} \frac{(-s)^{k}}{k !} B_{k},
$$

where

$$
B_{0}=\int_{\mathcal{M}} \sqrt{g}, \quad B_{1}=\left(\xi-\frac{1}{6}\right) \int_{\mathcal{M}} \sqrt{g} R,
$$

(with $\xi=\frac{1}{4} \frac{D-2}{D-1}$ ) and the general term has the structure

$$
\begin{aligned}
B_{k}=\int_{\mathcal{M}} \sqrt{g}\left[R \square^{k-2} R\right. & +\sum_{0 \leq i \leq 2 k-6} R \nabla^{i} R \nabla^{2 k-6-i} R+\ldots \\
& \left.+\sum_{0 \leq i \leq k-3} R^{i}(\nabla R) R^{k-i-3} \nabla R+R^{k}\right] .
\end{aligned}
$$

\footnotetext{
${ }^{8}$ When the metric has conical singularities the coefficients $B_{i}(i \geq 1)$ will acquire additional surface terms at the horizon which vanish as $T \rightarrow T_{H}$ [22]. Since we are not going to work with this formulation we will omit writing these out.
} 
On dimensional grounds, it is clear that only the $B_{0}$ term will give a bulk contribution which however diverges like $\epsilon^{-D}$. This term is independent of the temperature and does not contribute to the free energy nor to the entropy. It should be canceled against the bare cosmological constant in order that the original (e.g. Schwarzschild instanton) solution may be a valid saddle point. In the corresponding supersymmetric case this contribution would be zero. The entropy comes solely from the curvature terms in the determinant since a nontrivial $\beta$-dependence can arise only from the conical singularity $\int R \sim 2 \pi-\beta$ that is introduced when one moves away from the Hawking temperature in order to compute the entropy [18, 7, 8]. Since in Rindler space the curvature at the Hawking temperature is exactly zero, these $\beta$-dependent terms vanish at the Hawking temperature. Consequently the entire free energy of the gas of particles at the Hawking temperature must come from the Liouville-type action (or from non-local terms which are not modeled by the asymptotic expansion). However except in two dimensions the Liouville-type term does not appear to be infrared divergent. We must thus conclude that there is no bulk free energy in Rindler space or that it must arise from terms which do not occur in the asymptotic expansion. Furthermore in this method the origin of the bulk entropy of the gas of bosons (in any static background) is also obscure. Again the local terms occurring in the asymptotic expansion of the heat kernel cannot contribute to this bulk entropy since none of them (except the cosmological constant term which cannot contribute to the entropy) can have a cubic infrared divergence. On the other hand this contribution does not seem to come from the Liouville-type action term in the first line of (3.17) (see for instance the expression (3.16) for four dimensions). It must thus come from non-local terms in the determinant. In the case of Rindler space we will confirm (in sect. 5) from the optical method that both the free energy and entropy are proportional to the area, i.e. there is no bulk term.

\footnotetext{
${ }^{9}$ This observation has been made earlier by B. Allen [23] who considered the difference between the partition function for the normal ordered hamiltonian and the optical space [10] functional integral. However the relation between the partition function and optical method on the one hand, and the functional integral in the original metric on the other, was not discussed there.
} 
The presence of the Liouville-type term makes calculation of entropy using the original metric somewhat awkward. Since the Liouville-type action involves curvature terms (see (3.16)), there will be contributions from it to the entropy and a nontrivial $\beta$-dependence will be introduced through the conical singularities. However these terms will be finite so one can safely calculate all the divergent contributions to the entropy by ignoring the Liouville-type action.

Let us now turn to the optical space method. In the second line of (3.17), the relevant manifold has the topology of $S^{1} \times \mathcal{M}_{\mathrm{D}-1}$ and the heat kernel factorizes since $-\bar{K}_{\beta}=$ $\partial_{\omega}^{2}+\bar{\square}_{D-1}-\xi R$ and $\bar{\square}_{D-1}$ is independent of $\omega$. Thus we have

$$
-\beta F=\frac{1}{2} \int \frac{d s}{s} \int_{0}^{\beta} d \omega H_{S^{1}} \int \sqrt{\bar{g}} d^{D-1} x \bar{H}_{D-1}(s \mid x, x),
$$

where

$$
H_{S^{1}}=\beta^{-1} \sum_{n=-\infty}^{\infty} e^{-s\left(\frac{2 n \pi}{\beta}\right)^{2}}=\frac{1}{(4 \pi s)^{\frac{1}{2}}} \sum_{n=-\infty}^{\infty} e^{-\frac{\beta^{2} n^{2}}{4 s}}
$$

after using the Poisson resummation formula. In the second integral of (3.22), we may use the asymptotic expansion of the heat kernel given by (3.19)-(3.21) except that $\sqrt{g}$ is replaced by $\sqrt{\bar{g}}, e^{-s m^{2}}$ by $e^{-s \Omega^{-2} m^{2}}$ (which goes inside the integrals defining the $B$ 's), $R$ by $\bar{R}$ and $\mathcal{M}_{\mathcal{D}}$ by $\mathcal{M}_{\mathrm{D}-1} \cdot$ 四

Thus one gets from the optical point of view, the following expression for the free energy after subtracting the zero temperature cosmological constant term (i.e. the $n=0$ term in the thermal sum):

$$
F(\beta)=-\frac{1}{2} \int_{0}^{\infty} \frac{d s}{s} \frac{1}{(4 \pi s)^{\frac{D}{2}}} \sum_{n \neq 0} e^{-\frac{\beta^{2} n^{2}}{4 s}} \sum_{k=0}^{\infty} \frac{(-s)^{k}}{k !} \bar{B}_{k} .
$$

It is important to note that in this calculation the free energy indeed has as expected an ultraviolet divergence, but it comes from the divergence of the optical metric and not from the $s=0$ end of the proper time integral. In fact the only divergence comes from

\footnotetext{
${ }^{10}$ There are also $m$-dependent non-covariant terms coming from the non-commutativity of $\Omega$ and the Klein-Gordon operator. However we ignore them since we are interested only in the high-temperature limit where mass terms are irrelevant. We keep the mass-dependent exponential factor merely as an infrared regulator until we finally take the high-temperature limit.
} 
the divergence of the density $\sqrt{\bar{g}}$ at the horizon. To clarify this and several other issues we will look at the examples of Rindler and black hole spaces in the following sections. Before we do that, let us close this section by writing down the high-temperature limit of the above formula for the free energy. This is easily evaluated by first changing the variable of the proper time integral from $s$ to $u=\beta^{-2} s$ and then neglecting the higher powers of $\beta^{2}$ coming from the expansion in (3.24). Thus we get

$$
F(\beta)=-T^{D} \int \frac{d u}{u} \frac{1}{(4 \pi u)^{\frac{D}{2}}} \sum_{n=1}^{\infty} e^{-\frac{n^{2}}{4 u}} \int_{\mathcal{M}_{\mathrm{D}-1}} \sqrt{\bar{g}} e^{-\frac{\Omega^{-2} m^{2}}{T^{2}}} .
$$

Or if our system consists of only particles with masses that are small compared to the temperature (the above form is suitable for a generalization to string theory where this is certainly not the case), we get

$$
\begin{aligned}
F & =-T^{D} V_{D-1} \int_{0}^{\infty} \frac{d u}{u} \frac{1}{(4 \pi u)^{\frac{D}{2}}} \sum_{n=1}^{\infty} e^{-\frac{n^{2}}{4 u}} \\
& =-\frac{T^{D} V_{D-1}}{\pi^{\frac{D}{2}}} \Gamma\left(\frac{D}{2}\right) \zeta(D),
\end{aligned}
$$

where $V_{D-1}=\int_{\mathcal{M}_{\mathrm{D}-1}} \sqrt{\bar{g}}$ is the volume of optical space. This is just the free energy of a gas of (massless) particles in a box whose volume is given by the optical measure. Thus in four dimensions we have

$$
F=-\frac{\pi^{2}}{90} V_{3} T^{4}, \quad S=\frac{2 \pi^{2}}{45} V_{3} T^{3}
$$

and in two dimensions

$$
F=-\frac{\pi}{6} V_{1} T^{2}, \quad S=\frac{\pi}{3} V_{1} T .
$$

Calculating the free energy and hence the entropy of the scalar fields in different backgrounds is now just a triviality. One just plugs in the volume of optical space corresponding to each metric.

\section{Free Energy of Fermions}

In this section, let us briefly show how our above results are modified for Dirac (Majorana) fermions. This will be important especially when we consider supersymmetric case. 
The contribution of fermions was also discussed in Ref. [24], but our unified treatment is much simpler.

We introduce the vierbein fields of the form

$$
v_{\mu}^{\alpha}=\left[\begin{array}{cc}
v_{0}^{0} & 0 \\
0 & w_{i}^{a}
\end{array}\right], \quad v_{\alpha}^{\mu}=\left[\begin{array}{cc}
\left(v_{0}^{0}\right)^{-1} & 0 \\
0 & w_{a}^{i}
\end{array}\right],
$$

where $\mu=0,1, \cdots, D-1 ; i=1,2, \cdots, D-1$ are curved indices and $\alpha=0,1, \cdots, D-$ $1 ; a=1,2, \cdots, D-1$ are tangent space ones. The action is

$$
\begin{aligned}
S & =\int d^{D} x v\left(i \bar{\psi} \gamma^{\alpha} v_{\alpha}^{\mu} \nabla_{\mu} \psi-m \bar{\psi} \psi\right) \\
& =\int d t \int d^{D-1} x w\left(i \psi^{\dagger} \nabla_{0} \psi+i \bar{\psi} \gamma^{a} v_{0}^{0} w_{a}^{i} \nabla_{i} \psi-m v_{0}^{0} \bar{\psi} \psi\right),
\end{aligned}
$$

where $v=\operatorname{det} v_{\mu}^{\alpha}=v_{0}^{0} w, w=\operatorname{det} w_{i}^{a}, \nabla_{\mu}=\partial_{\mu}+\Gamma_{\mu}$ with the connection $\Gamma_{\mu}=$ $\frac{1}{2} \Sigma^{\alpha \beta} v_{\alpha}^{\nu} \frac{\partial}{\partial x^{\mu}} v_{\beta \nu}, \Sigma^{\alpha \beta}$ being the generator of the Lorentz group for a Dirac field.

The canonical momenta are $\pi_{\psi}=i \psi^{*}$ and the Hamiltonian is

$$
H=\int d^{D-1} x \mathcal{H}=\int d^{D-1} x v\left(-i \psi^{\dagger}\left(v_{0}^{0}\right)^{-1} \Gamma_{0} \psi-i \bar{\psi} \gamma^{a} w_{a}^{i} \nabla_{i} \psi+m \bar{\psi} \psi\right) .
$$

Introducing the time-slicing procedure to define path integral, as in the scalar fields, we find the partition function is given by

$$
\operatorname{Tr}\left[e^{-\beta H}\right]=\int_{\psi(0, \vec{x})=-\psi(\beta, \vec{x})} \prod_{t, \vec{x}}\left(d \psi d \psi^{*} w\right) e^{-\int_{0}^{\beta} d t \int d^{D-1} x v \mathcal{L}}
$$

where

$$
\mathcal{L}=\left(i \bar{\psi} \gamma^{\alpha} v_{\alpha}^{\mu} \nabla_{\mu} \psi-m \bar{\psi} \psi\right)
$$

Introducing the optical vierbein

$$
\tilde{v}_{\mu}^{\alpha}=\frac{v_{\mu}^{\alpha}}{v_{0}^{0}} \equiv \Omega v_{\mu}^{\alpha}
$$

and $\tilde{\psi}=\Omega^{(1-D) / 2} \psi\left(\tilde{\psi}^{*}=\Omega^{(1-D) / 2} \psi^{*}\right)$, we find

$$
\begin{aligned}
\operatorname{Tr}\left[e^{-\beta H}\right] & =\int \prod_{t, \vec{x}}\left(\tilde{v} d \tilde{\psi} d \tilde{\psi}^{*}\right) e^{-\int_{0}^{\beta} d t \int d^{D-1} x \tilde{v} \mathcal{L}}, \\
& =\int \prod_{t, \vec{x}}\left(v d \psi d \psi^{*}\right) e^{-\int_{0}^{\beta} d t \int d^{D-1} x v \mathcal{L}+\Gamma_{F}},
\end{aligned}
$$

\footnotetext{
${ }^{11}$ Here we ignore the mass terms for simplicity. It is easy to recover them in the following formulae and in any case they drop out in our high-temperature approximation.
} 
where again $\Gamma_{F}$ is a Liouville-type action for fermions.

We calculate the functional determinant resulting from the first line of (4.7) using the heat kernel. The result can be expressed as

$$
\beta F=\frac{1}{2} \zeta_{F}^{\prime}(0)
$$

where

$$
\zeta_{F}(p)=\sum_{n} \frac{1}{\Gamma(p)} \int_{0}^{\infty} d s s^{p-1} \int d^{D-1} x \sqrt{\bar{g}}\left\langle x\left|e^{-s \bar{K}_{F}}\right| x\right\rangle,
$$

with $\bar{K}_{F}$ is the Feynman propagator in the optical vierbein. Noting the antiperiodic conditions for fermions and using the Poisson resummation formula, we get

$$
\zeta_{F}(p)=\frac{\beta}{\Gamma(p)} \int_{0}^{\infty} d s s^{p-1} \frac{1}{(4 \pi s)^{\frac{D}{2}}} \sum_{n=-\infty}^{\infty}(-1)^{n} e^{\frac{\beta^{2} n^{2}}{4 s}} \sum_{k=0}^{\infty} \frac{(-t)^{k}}{k !} \bar{B}_{k}^{(F)},
$$

where use has also been made of the asymptotic expansion of the heat kernel for fermions. The coefficients $\bar{B}_{k}^{(F)}$ are given by (see for example [19])

$$
\bar{B}_{0}^{(F)}=f \int_{\mathcal{M}} \sqrt{\bar{g}}, \bar{B}_{1}^{(F)}=-\frac{f}{12} \int_{\mathcal{M}} \sqrt{\bar{g}} \bar{R}
$$

etc. with $f=2^{[D / 2]}$ being the number of spinor components in $D$ dimensions. Thus after dropping the $n=0$ term which can be absorbed into the zero-temperature cosmological constant (in a supersymmetric case it will cancel the bosonic contribution), we finally obtain the free energy

$$
F(\beta)=\frac{1}{2} \int_{0}^{\infty} \frac{d s}{s} \frac{1}{(4 \pi s)^{\frac{D}{2}}} \sum_{n \neq 0}(-1)^{n} e^{-\frac{\beta^{2} n^{2}}{4 s}} \sum_{k=0}^{\infty} \frac{(-s)^{k}}{k !} \bar{B}_{k}^{(F)},
$$

Note the close similarity to eq. (3.24) for scalar field.

In the high-temperature limit, we can use a similar procedure for scalar fields to obtain

$$
\begin{aligned}
F & =T^{D} V_{D-1} \int_{0}^{\infty} \frac{d u}{u} \frac{f}{(4 \pi u)^{\frac{D}{2}}} \sum_{n=1}^{\infty}(-1)^{n} e^{-\frac{n^{2}}{4 u}} \\
& =-2^{[D / 2]}\left(1-2^{1-D}\right) \frac{T^{D} V_{D-1}}{\pi^{\frac{D}{2}}} \Gamma\left(\frac{D}{2}\right) \zeta(D) .
\end{aligned}
$$

where $V_{D-1}$ is again the volume of optical space. 
In four dimensions we have

$$
F=-\frac{7}{2} \frac{\pi^{2}}{90} V_{3} T^{4}, \quad S=\frac{7 \pi^{2}}{45} V_{3} T^{3}
$$

and in two dimensions

$$
F=-\frac{\pi}{6} V_{1} T^{2}, \quad S=\frac{\pi}{3} V_{1} T .
$$

If the fermions are Majorana, these get an additional factor $1 / 2$.

In the following sections we will apply these formulae to the examples of Rindler and black hole spaces.

\section{Rindler Space}

Euclidean Rindler space has the metric $d s^{2}=R^{2} d \omega^{2}+d R^{2}+d x_{\perp}^{2}$. To avoid a conical singularity at the origin $\omega$ must be identified with period $\beta=2 \pi$ corresponding to a

temperature $T=\frac{1}{2 \pi}$. The logarithm of the partition function should then give us the free energy of a gas of bosons at this temperature (multiplied by $-2 \pi$ ). If one used the covariant functional integral to evaluate the partition function, one can just transform from polar coordinates to cartesian coordinates to demonstrate that the free energy is zero. Of course the functional integral will have the usual divergence associated with the zero temperature cosmological constant which must be canceled against the bare constant. Moreover in the supersymmetric case, this will be strictly zero though at any finite temperature one must get a non-zero free energy due to the different boundary conditions on bosons and fermions. Thus the path integral with the original metric does not give the right free energy, and the reason is that the correct path integral for the evaluation of the thermal ensemble is $(3.12)$ and not the one with the original metric. On the other hand, in the evaluation of the entropy one needs to go away from the Rindler temperature, thus introducing a conical singularity at the origin [18, 7, 8] when working with the original metric. The value of the entropy agrees with that computed in the thermal ensemble. As we will see (at least in two dimensions), this curiosity can be explained precisely in terms of the well-known Polyakov term. 
Rindler space (with $\beta=2 \pi$ ) is flat but the corresponding optical space which has metric $\overline{d s^{2}}=d \omega^{2}+\frac{1}{R^{2}}\left(d R^{2}+d x_{\perp}^{2}\right)$ has catuuuure $\overline{\mathcal{R}}=-(D-1)(D-2)$ which however vanishes for $D=2$. Thus in this case we have for the thermal partition function the expression (we will set $m=0$ for simplicity. It is irrelevant anyway in the high-temperature limit):

$$
\begin{aligned}
\operatorname{Tr}\left[e^{-\beta H}\right] & =\int_{\bar{\phi}(0, \vec{x})=\bar{\phi}(\beta, \vec{x})} \prod_{\omega, \vec{x}} d \bar{\phi} \bar{g}^{\frac{1}{4}}(\omega, \vec{x}) e^{-\int_{0}^{\beta} d \omega d^{D-1} x \sqrt{\bar{g}} \bar{g}^{\mu \nu} \partial_{\mu} \bar{\phi} \partial \nu \bar{\phi}} \\
& =\int_{\phi(0, \vec{x})=\phi(\beta, \vec{x})} \prod_{\omega, \vec{x}} d \phi g^{\frac{1}{4}}(\omega, \vec{x}) e^{-\int_{0}^{\beta} d \omega d R \sqrt{g} g^{\mu \nu} \partial_{\mu} \phi \partial \nu \phi+\Gamma} .
\end{aligned}
$$

In the above $\Gamma=\frac{1}{48 \pi} \int d^{2} x \sqrt{g} \frac{1}{2} g^{\mu \nu} \partial_{\mu} \ln \Omega^{2} \partial_{\nu} \ln \Omega^{2}$ is the Liouville action coming from the two-dimensional conformal anomaly. Let us first use the second line in the above equation to evaluate this. For $2 D$ Rindler space $\Omega=\frac{1}{R}$. Hence since the scalar field action gives no contribution to the free energy at $\beta=2 \pi$, we have from the Liouville action $\square$

$$
-2 \pi F=\frac{1}{48 \pi} \int_{0}^{2 \pi} d \omega \int_{\epsilon}^{L} R d R \frac{1}{R^{2}}
$$

where we have regulated the integral by introducing cutoffs at both short and long distances. Thus we get a free energy

$$
F=-\frac{1}{24 \pi} \ln \frac{L}{\epsilon}
$$

for a single scalar field, whereas for a Dirac spinor similar procedure yields the same result. (If the fermion is Majorana, the result is half this value.) This is in agreement with the direct Hamiltonian calculation of the free energy of a 1-dimensional gas of free bosons at a temperature $2 \pi$. To obtain the entropy one has to differentiate with respect to $\beta$ and going away from the Rindler value $\beta=2 \pi$ introduces a conical singularity which gives (from the curvature terms in the evaluation of the determinant) a $\beta$-dependence. There is no contribution to the entropy from the Liouville term since it gives only a $\beta$-independent contribution to $F$.

\footnotetext{
${ }^{12}$ For a related calculation see [25].
} 
It is instructive to look at the calculation also from the optical space path integral (first line of (5.1)). In this case after changing the spatial variable from $R$ to $x=\ln R$, we have

$$
e^{-\beta F}=\int_{\phi(0, \vec{x})=\phi(\beta, \vec{x})} \prod_{\omega, \vec{x}} d \phi(\omega, \vec{x}) e^{-\int_{0}^{\beta} d \omega \int_{-\infty}^{\infty} d x \bar{\phi}\left(\partial_{\omega}^{2}+\partial_{x}^{2}\right) \bar{\phi}} .
$$

This is the usual path integral form for the partition function for a gas of free bosons at an inverse temperature $\beta$. Indeed, from eq. (3.28) and eq. (4.15), we get (5.3) for $T=1 / 2 \pi$. Thus the optical metric calculation directly gives us the correct expression for the free energy as well as the entropy of Rindler space.

Rindler space is more complicated for $D>2$ dimensions. The partition function is now given by (reintroducing the mass term)

$$
\begin{aligned}
& \int_{\bar{\phi}(0, \vec{x})=\bar{\phi}(\beta, \vec{x})} \prod_{\omega, \vec{x}} d \bar{\phi} \bar{g}^{\frac{1}{4}}(\omega, \vec{x}) e^{-\int_{0}^{\beta} d \omega d^{D-1} x \sqrt{\bar{g}}\left[g^{\bar{\mu} \nu} \partial_{\mu} \bar{\phi} \partial_{\nu} \bar{\phi}-\frac{1}{4}(D-2)^{2} \bar{\phi}^{2}+m^{2} R^{2} \bar{\phi}^{2}\right]} \\
& =\operatorname{det}^{-\frac{1}{2}}\left[-\bar{\square}-\frac{1}{4}(D-2)^{2}+m^{2} R^{2}\right]
\end{aligned}
$$

where $\bar{\square}=\partial_{\omega}^{2}+R^{D-1} \partial_{R} \frac{R^{2}}{R^{D-1}} \partial_{R}+R^{2} \partial_{\perp}^{2}$. There is no purely spatial transformation of coordinates that will bring this into the form of a partition function for a gas of free bosons at the inverse temperature $\beta$. The second term in (5.5) arises from the conformal coupling due to the fact that the curvature in the optical space is non-zero. Thus all the terms in the asymptotic expansion in (3.24) will contribute. However, in the hightemperature approximation we will have (3.26) with

$$
V_{D-1}=V_{D-2} \int_{\epsilon}^{\infty} \frac{d R}{R^{D-1}}=\frac{V_{D-2}}{(D-2) \epsilon^{D-2}} .
$$

The last integral is divergent at the origin of optical space and in the high-temperature approximation; what we have is exactly the free energy of a gas of massless bosons in a spatial box endowed with the optical metric, i.e. the optical volume of the box is $\frac{V_{D-2}}{\epsilon^{D-2}}$. This expression for the free energy agrees with the first calculation done by Susskind and Uglum [7]. However we have ignored the terms coming from the (non-zero) optical space curvature $\left(B_{k}, k \neq 0\right)$ by taking the high-temperature approximation. These correspond to the contributions also neglected in the WKB approximation to calculate eigenvalues 
and in replacing the sum over modes by an integral in [7]. Alternatively they must correspond, in the functional integral calculation of [7] in the original metric to finite non-zero contributions coming from the terms which are of higher order in curvatures. [3]

\section{Black Hole Backgrounds}

The four-dimensional Schwarzschild black hole has the metric (setting $G_{N}=1$ )

$$
d s^{2}=-\left(1-\frac{2 M}{r}\right) d t^{2}+\frac{d r^{2}}{\left(1-\frac{2 M}{r}\right)}+r^{2} d \Omega_{2} .
$$

The corresponding optical volume is

$$
\begin{aligned}
V_{3}^{S c h} & =4 \pi \int_{2 M+\epsilon}^{R} \frac{r^{2}}{\left(1-\frac{2 M}{r}\right)^{2}} \\
& =4 \pi\left(\frac{R^{3}}{3}+2 M R^{2}+12 M^{2} R+32 M^{3} \ln \frac{R-2 M}{\epsilon}\right. \\
& \left.+\frac{16 M^{4}}{\epsilon}-\frac{104 M^{3}}{3}+O\left(R^{-1}\right)+O(\epsilon)\right)
\end{aligned}
$$

By plugging this into (3.26) and (4.13), we immediately get the (quantum corrections to) the free energy and hence the entropy of the black hole in the high-temperature approximation. Here we see the divergence first observed by [9]. Although it appears linear in terms of the coordinate cutoff $\epsilon$, it is quadratic in terms of the proper distance cutoff $\delta=\sqrt{2 M \epsilon}$ in the Schwarzschild geometry. We also see another logarithmic divergence. These additional divergences were first discovered by working with the functional integral in the original metric (3.9). However in that case the calculation is much more complicated [14].

Next let us consider the Reissner-Nordström charged black hole. This example is interesting because it has an extreme limit when the mass becomes equal to the charge.

\footnotetext{
${ }^{13}$ There is a formal argument given in [7] to the effect that these terms must vanish. However it involves delicate issues arising from having products of conical singularities (delta functions). Indeed it has been argued in [26] on somewhat different grounds than the above, that these terms must contribute to the entropy.
} 
The metric is

$$
d s^{2}=-\left(1-\frac{2 M}{r}+\frac{Q^{2}}{r^{2}}\right) d t^{2}+\left(1-\frac{2 M}{r}+\frac{Q^{2}}{r^{2}}\right)^{-1} d r^{2}+r^{2} d \Omega_{2}
$$

This black hole has an ADM mass $M$ and an electric charge $Q$. The metric has outer and inner horizons at

$$
r_{ \pm}=M \pm\left(M^{2}-Q^{2}\right)^{\frac{1}{2}}
$$

In order to avoid a naked singularity we must have $M \geq Q$. The Hawking temperature of this hole is given by $T=\frac{\left(r_{+}-r_{-}\right)}{4 \pi r_{+}^{2}}$ (which goes to zero as $M \rightarrow Q$ ) and the entropy is again given by the quarter the area of the horizon $S=\frac{1}{4} 4 \pi r_{+}^{2}$ as in the Schwarzschild case. In the limit $M \rightarrow Q$, the two horizons become degenerate and the metric of this extreme hole is

$$
d s^{2}=-\left(1-\frac{M}{r}\right)^{2} d t^{2}+\frac{d r^{2}}{\left(1-\frac{M}{r}\right)^{2}}+r^{2} d \Omega_{2} .
$$

Although the limiting temperature of the Reissner-Nordström black hole in the extreme limit is zero and its entropy is $\pi M^{2}$, from purely geometrical considerations in the above metric, it seems that the temperature is arbitrary and that its entropy is zero [27 even though the area of the horizon is non-zero. This seems to be rather puzzling from the thermodynamic point of view. We will see below that the calculation of the contribution of the scalar fields to the entropy sheds some light on this issue.

For the non-degenerate case the optical volume is

$$
\begin{aligned}
V_{3}^{r n} & =4 \pi \int_{r_{+}+\epsilon}^{R} \frac{r^{6} d r}{\left(r-r_{+}\right)^{2}\left(r-r_{-}\right)^{2}}=4 \pi\left[\frac{R^{3}}{3}+2 M R^{2}+\left(3 r_{+}^{2}+4 r_{+} r_{-}+3 r_{-}^{2}\right) R\right. \\
& +\frac{r_{+}^{6}}{\left(r_{+}-r_{-}\right)^{2} \epsilon}+\frac{r_{-}^{6}}{\left(r_{+}-r_{-}\right)^{3}}+\frac{2 r_{+}^{5}\left(2 r_{+}-3 r_{-}\right)}{\left(r_{+}-r_{-}\right)^{3}} \ln \frac{R-r_{+}}{\epsilon} \\
& +\frac{2 r_{-}^{5}\left(3 r_{+}-2 r_{-}\right)}{\left(r_{+}-r_{-}\right)^{3}} \ln \frac{R-r_{-}}{r_{+}-r_{-}}-r_{+}\left(\frac{13}{3} r_{+}^{2}+5 r_{+} r_{-}+3 r_{-}^{2}\right) \\
& \left.+O\left(R^{-1}\right)+O(\epsilon)\right] .
\end{aligned}
$$

Substituting this in (3.26) or (4.13), we get the expressions for the (quantum corrections to the) free energy and hence also the entropy in this space. The leading divergence is

\footnotetext{
${ }^{14}$ We wish to thank L. Susskind for pointing this out.
} 
again linear (or quadratic in the proper cutoff) and there is an additional logarithmic divergence. However, we also see the appearance of inverse powers of the difference in the two horizon radii. This clearly implies that the extreme limit is very singular. Indeed this is confirmed by a direct calculation of free energy and entropy for the extreme black hole. From (6.5), we have for the optical volume

$$
\begin{aligned}
V_{3}^{e x t}= & \int_{M+\epsilon}^{R} \frac{r^{6} d r}{(r-M)^{4}}=4 \pi\left[\frac{R^{3}}{3}+2 M R^{2}+10 M^{2} R+\frac{M^{6}}{3 \epsilon^{3}}+\frac{3 M^{5}}{\epsilon^{2}}\right. \\
& \left.+\frac{15 M^{4}}{\epsilon}+20 M^{3} \ln \frac{R-M}{\epsilon}-\frac{37}{3} M^{3}+O\left(R^{-1}\right)+O(\epsilon)\right] .
\end{aligned}
$$

Here we see the appearance of cubic and quadratic divergences. Clearly the thermodynamics of the extreme limit is not well-defined since, although the linear and logarithmic divergences may be absorbed into the renormalization of $G_{N}$ [7] and the coefficients of higher powers of curvature in the expansion of the effective action, this will not be the case for these higher order divergences. We suggest therefore that the thermodynamics of the extreme limit of the Reissner-Nordström black hole is not well-defined. In fact it would seem that in any discussion of thermal properties the extreme black hole (which should be able to absorb a quantum and become non-extreme) must necessarily be treated as the limiting case of the non-extreme Reissner-Nordström black hole.

Finally we discuss dilaton black holes [29]. The metric is given in this case by

$$
d s^{2}=-\left(1-\frac{2 M}{r}\right) d t^{2}+\frac{d r^{2}}{\left(1-\frac{2 M}{r}\right)}+r(r-a) d \Omega_{2},
$$

where $a$ is a constant. The corresponding optical volume is

$$
\begin{aligned}
V_{3} & =4 \pi \int_{2 M+\epsilon}^{R} \frac{r(r-a)}{\left(1-\frac{2 M}{r}\right)^{2}} d r \\
& =4 \pi\left(\frac{R^{3}}{3}+\left(2 M-\frac{a}{2}\right) R^{2}+4 M(3 M-a) R+\frac{8 M^{3}(2 M-a)}{\epsilon}\right. \\
& \left.+4 M^{2}(8 M-3 a) \ln \frac{R-2 M}{\epsilon}-M^{2}\left(\frac{104 M}{3}-10 a\right)+O\left(R^{-1}\right)+O(\epsilon)\right) .
\end{aligned}
$$

\footnotetext{
${ }^{15}$ It has been claimed [28] that with Pauli Villars regulators the entropy of the extreme black hole is no more singular than that of the non-extreme one. However the authors go on to point out that the temperature still needs to be taken to be zero which is in agreement with our conclusion above.
} 
As in the Schwarzschild case here too there is a linear as well as a logarithmic divergence and again one may argue following [7] that the former can be absorbed in a renormalization of $G_{N}$. In the extreme limit $\left(M \rightarrow \frac{a}{2}\right)$, the "classical" entropy $\left(S_{c l}=\frac{A}{4}=2 \pi M(2 M-a)[30]\right)$ vanishes and so does the linear divergence. However the logarithmic divergence remains.

\section{Conclusions}

In this paper we have shown that the thermal partition function is directly related to the functional integral in the so-called optical metric, and that this differs from the determinant of the Laplacian in the original metric by a Liouville-type term. As a consequence we find that the conical singularity method calculates correctly only the divergent parts of the entropy. It should be pointed out that the thermodynamic entropy that we have discussed is not related in any direct way to the entropy of entanglement (quantum entropy) that needs to be considered in order to discuss the issue of whether quantum mechanical information is lost in the process of black hole evaporation. This effect (or its absence) is tied up with deep issues in quantum gravity such as the problem of time and probably also the interpretation of quantum mechanics itself. What we have studied is the standard thermostatistical question of counting the number of accessible micro-states for a macroscopically specified system, in this case the mass (and charge for the Reissner-Nordström case) of a black hole. We have found the quadratic divergence in this entropy that was found previously but in addition we found a logarithmic divergence

which cannot be interpreted as a renormalization of Newton's constant. One may of course subtract this by introducing a $R^{2}$ counterterm into the original gravity action 14, 22]. Similar results are valid for both non-extreme charged black holes and for dilatonic black holes. However in the extreme charged black hole case we have observed that although the limiting case seems to have no particular problems the thermodynamics of the limit itself (considered in isolation i.e. not as the limit of the non-extreme case) does not have well-defined thermal properties. This is already evident at the classical 
level and is exacerbated at the quantum level.

Although it is not possible to understand the issue of quantum information loss without resolving the deep issues we mentioned above, one may still hope to understand the operation of the second law of thermodynamics in this system. Unfortunately a full discussion of this would involve a solution of a time evolving system and that is beyond our scope at present. One can however discuss the stability of the equilibrium between the bath of radiation and the black hole following [10] and other authors. black hole entropy was taken to be the classical one and the entropy of the radiation was taken to be the standard thermodynamic formula. In this work we have obtained from one calculation both the black hole entropy with quantum corrections as well as the bulk entropy of the radiation. After absorbing the divergences by introducing counterterms into the classical gravitational action (as well as a $R^{2}$ term) the same qualitative features will be obtained (although naturally there will be quantitative differences). Namely the black hole will be in stable equilibrium with the radiation bath provided the volume (determined by our infrared cutoff $R$ in the previous section) is less than a critical value. The calculation using the conical singularity method, on the other hand, will not easily yield the (bulk) contribution of the radiation to the entropy (i.e. a $R^{3} T^{3}$ piece) and thus cannot by itself (i.e. ignoring the Liouville-type term and possible non-local terms) satisfy the second law.

\section{Acknowledgments}

SdeA would like to acknowledge the award of a Japan Society for the Promotion of Science fellowship, and the hospitality of Profs. K. Higashijima and E. Takasugi at Osaka University where this investigation was begun. He would also like to thank Gary Gibbons and Lenny Susskind for discussions on extremal black holes and entropy, and Doug MacIntire for discussions on this work. This work is partially supported by the Department of Energy contract No. DE-FG02-91-ER-40672.

\footnotetext{
${ }^{16}$ For a nice discussion of these issues and for references to earlier literature, see [31].
} 


\section{References}

[1] S. Hawking, Comm. Math. Phys. 43, 199 (1975).

[2] J. Bekenstein, Phys. Rev. D7, 2333 (1973); D9, 3292 (1974).

[3] G. Gibbons and S. Hawking, Phys. Rev. D15, 2752 (1977); S.W. Hawking, in "General Relativity, an Einstein centenary survey", S.W. Hawking and W. Israel, (eds.) Cambridge 1979; G. Horowitz and S.W. Hawking, preprint (1995) gr-qc/9501014.

[4] W. Zurek and K.S. Thorne, Phys. Rev. Lett. 54, 2171 (1985).

[5] L. Bombelli, R. Koul, J. Lee and R. Sorkin, Phys. Rev. D34, 373 (1986).

[6] M. Sredinicki, Phys. Rev. Lett. 71, 666 (1993).

[7] L. Susskind and J. Uglum, Phys. Rev. D50, 2700 (1994).

[8] C. Callan and F. Wilczek, Phys. Lett. B333, 55 (1994); D. Kabat and M. J. Strassler, Phys. Lett. B329, 46 (1994), D. Kabat preprint RU-95-06, hep-th/9503016.

[9] G. 't Hooft, Nucl. Phys. B256, 727 (1985).

[10] G. Gibbons and M. Perry, Proc. R. Soc. Lond. A358, 467 (1978).

[11] J.S. Dowker and G. Kennedy, J. Phys. A11, 895 (1978).

[12] J. L. F. Barbon, Phys. Rev. D50, 2712 (1994).

[13] R. Emparan, preprint EHU-FT-94/5, hep-th/9407064.

[14] S. N. Solodukhin, Phys. Rev. D51, 618 (1995).

[15] S.P. de Alwis and N. Ohta, preprint COLO-HEP-347, OU-HET 207, hep-th/9412027.

[16] A. Ghosh and P. Mitra, Phys. Rev. Lett. 73, 2521 (1994) and preprint SISSA-17294-EP, hep-th/9411128. 
[17] J. D. Brown and J.W. York, Phys. Rev. D471420 (1992).

[18] C. Bañados, C. Teitelboim, and J. Zanelli, Phys. Rev. Lett. 72, 957 (1994); C. Teitelboim, preprint hep-th/9410103.

[19] N. D. Birrell and P. C. W. Davies, Quantum fields in curved space, Cambridge Univ. Press, 1982.

[20] A. M. Polyakov, Phys. Lett. B103, 207 (1981).

[21] R. J. Riegert, Phys. Lett. B134, 56 (1984); E. S. Fradkin and A. A. Tseytlin, Phys. Lett. B134, 187 (1984); E. T. Tomboulis; Nucl. Phys. B329, 410 (1990); S. Odintsov and I. Shapiro, Class. Quant. Grav. 9, 873 (1992); I. Antoniadis, P.O. Mazur, and E. Mottola, Nucl. Phys. B388, 627 (1992).

[22] D. V. Fursaev and S. N. Solodukhin, preprints hep-th/9412020, and hep-th/9501127.

[23] B. Allen, Phys. Rev. D33 3640 (1986)

[24] L. Finn and F. Wilczek, preprint PUPT-1480, hep-th/9408089 (1994).

[25] S. Giddings, Phys. Rev. D49, 4078 (1993).

[26] V. Frolov and I. Novikov, Phys. Rev. D48, 4545 (1993).

[27] G.W. Gibbons, in Fields and Geometry, proceedings of 22nd Karpacz Winter School 1986, ed. A. Jadczyk (World Scientific, 1986), H. F. Dowker, J. Gauntlett, S. B. Giddings,and G. Horowitz, Phys. Rev. D50 2662 (1994), S. Hawking, G. Horowitz and S. Ross, preprint NI-94-012, gr-qc/9409013, and C. Teitelboim in reference 18.

[28] J-G Demers, R. Lafrance, and R. Myers, prepreint McGill/95-06, gr-qc/9503003.

[29] G. Gibbons and K. Maeda, Nucl. Phys. B298, 741 (1988); D. Garfinkle, G. Horowitz, and A. Strominger, Phys. Rev. D43, 3140; D45, 3888 (E) (1992). 
[30] J. Preskill, P. Schwarz, A. Shapere, S. Trivedi, and F. Wilczek, Mod. Phys. Lett. A6, 2353 (1991).

[31] K.S. Thorne, W. Zurek, R. H. Price, in "Black holes and the membrane paradigm" pages 320-324; Yale (1986). 